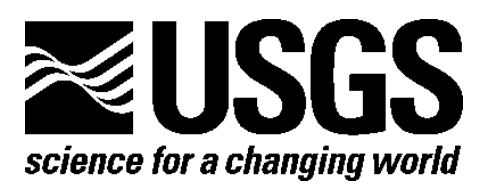

\title{
Lead Isotope Database of Unpublished Results from Sulfide Mineral Occurrences-California, Idaho, Oregon, and Washington
}

By S. E. Church

Open-File Report 2010-1157

U.S. Department of the Interior

U.S. Geological Survey 


\section{U.S. Department of the Interior \\ KEN SALAZAR, Secretary}

\section{U.S. Geological Survey \\ Marcia K. McNutt, Director}

U.S. Geological Survey, Reston, Virginia: 2010

For product and ordering information:

World Wide Web: http://www.usgs.gov/pubprod

Telephone: 1-888-ASK-USGS

For more information on the USGS—-the Federal source for science about the Earth, its natural and living resources, natural hazards, and the environment:

World Wide Web: http://www.usgs.gov

Telephone: 1-888-ASK-USGS

Suggested citation:

Church, S.E., 2010, Lead isotope database of unpublished results from sulfide mineral occurrences-California, Idaho, Oregon, and Washington: U. S. Geological Survey Open-File Report 2010-1157, 9 p.

Any use of trade, product, or firm names is for descriptive purposes only and does not imply endorsement by the U.S. Government.

Although this report is in the public domain, permission must be secured from the individual copyright owners to reproduce any copyrighted material contained within this report. 


\section{Contents}

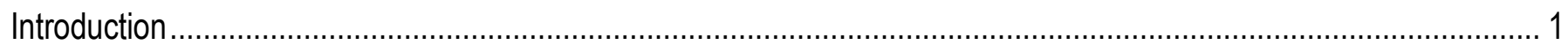

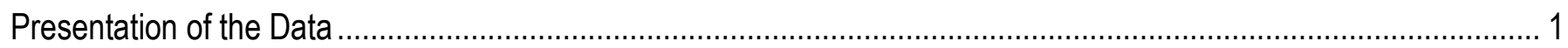

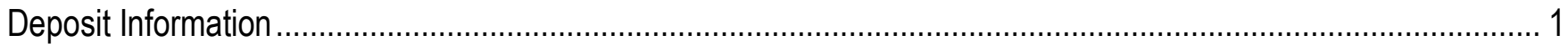

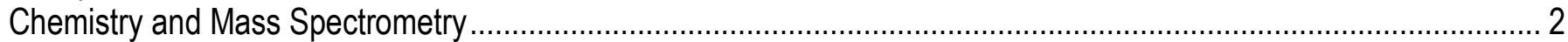

Mass Spectrometer

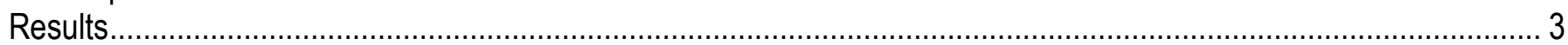

Acknowledgments

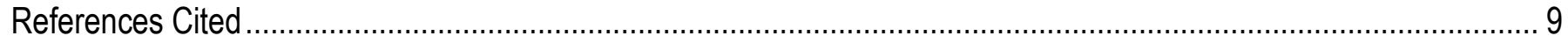

\section{Tables}

1. Compilation of geologic, mineral deposit type, sample mineralogy, $\mathrm{U}, \mathrm{Th}$, and $\mathrm{Pb}$ concentrations, and $\mathrm{Pb}$ isotope data for samples from northern Washington.

2. Compilation of geologic, mineral deposit type, sample mineralogy, $\mathrm{U}, \mathrm{Th}$, and $\mathrm{Pb}$ concentrations, and $\mathrm{Pb}$ isotope data for samples from Blue Mountains, Oregon and Idaho.

3. Compilation of geologic, mineral deposit type, sample mineralogy, $\mathrm{U}, \mathrm{Th}$, and $\mathrm{Pb}$ concentrations, and $\mathrm{Pb}$ isotope data for samples from Klamath Mountains, western Oregon and northern California

4. Compilation of geologic, mineral deposit type, sample mineralogy, $\mathrm{U}, \mathrm{Th}$, and $\mathrm{Pb}$ concentrations, and $\mathrm{Pb}$ isotope data for samples from Shasta mining district and California foothills belt, California

5. Abbreviations used in tables $1-4$ 


\title{
Lead Isotope Database of Unpublished Results from Sulfide Mineral Occurrences-California, Idaho, Oregon, and Washington
}

\author{
By S.E. Church
}

\section{Introduction}

$\mathrm{The} \mathrm{Pb}$ isotope database for sulfide deposits and occurrences in the Western United States was funded by the Mineral Resources Program, U.S. Geological Survey (USGS). Reports on Pb isotope data from Alaska were published in Church and others (1987a) and Gaccetta and Church (1989). The primary objectives of the project were three-fold:

- To utilize $\mathrm{Pb}$ isotope signatures, in conjunction with the regional mapping, to assess the relative ages and to categorize the types of deposits studied,

- To relate the $\mathrm{Pb}$ isotope and trace-element geochemical signatures of specific deposits and occurrences to ore-forming processes, and

- To use the $\mathrm{Pb}$ isotope data to correlate lithotectonic terranes within the northern Cordillera.

The report by Church and others (1987b) shows how this fingerprinting methodology can be applied to trace the offset of lithostratigraphic terranes.

\section{Presentation of the Data}

The $\mathrm{Pb}$ isotopic data presented in tables 1-4 represent the work completed on sulfide mineral deposits located in California, Oregon, Idaho, and Washington from 1986 through 1995 when the project was terminated due to reductions in funding. The data are reported here for use by investigators who may find them to be of value in mineral exploration. No attempt will be made in this report to summarize the voluminous literature on these ore deposits.

\section{Deposit Information}

Geologic and site information on each specific deposit or occurrence has been provided largely by the sample contributor on the form used for sample submission. All sites have been evaluated by comparing the data provided against the data in the USGS Mineral Resource Data System (MRDS; accessed June 29, 2010; http://tin.er.usgs.gov/mrds/). Specific geologic information on the deposit or occurrence also has been obtained from published literature referenced therein. Contributors were given the opportunity to modify the descriptive data in the tables. This process should have minimized errors. The deposit classification used in this report is based on the compilation by Cox and Singer (1986) and is included here only for the purpose of dialogue. There is not widespread agreement among geologists on the classification of deposits into model types. 
Since some of the samples have been obtained from museums (see table 5 for identification criteria), the localities of the samples may have a higher level of uncertainty associated with them. Some additional caution should be applied when using data from museum samples.

\section{Chemistry and Mass Spectrometry}

All of the $\mathrm{Pb}$ isotope data presented in tables 1-4 have been analyzed using the silica-gel emitter method (Cameron and others, 1969). Terms used in the data tables are defined in table 5. The $\mathrm{Pb}$ isotope data have all been corrected for thermal fractionation using the NBS SRM-981 common-Pb standard (Catanzaro and others, 1968) and are accurate, at the 2 sigma level, to within \pm 0.1 percent or better.

We report analyses from two types of samples: analyses made on those that contain galena $(\mathrm{PbS}$, indicated by Gn in the sample mineralogy column) or tetrahedrite (TT) that could be hand separated, and analyses on either mixed sulfides. Lead isotopic determinations have been made largely on mixed sulfides. Where mixed sulfides have been analyzed, we have given the $\mathrm{Pb}$ concentration in the sample determined either by direct-current-arc emission spectrography or by atomic absorption spectrophotometry in the solution used for $\mathrm{Pb}$ isotopic analysis. Previous studies of mixed sulfides, or of separate sulfide minerals that have $100 \mathrm{ppm}$ or more of $\mathrm{Pb}$, indicate that the $\mathrm{Pb}$ isotopic data obtained from this type of sample were comparable to those obtained from galena (for example, Church and others, 1986; Gulson, 1986).

Several different chemical procedures have been used on special samples analyzed in this study. In general, galena has been hand-picked for analysis where possible. Galena samples were prepared for analysis by digestion with ultrapure warm $16 \mathrm{M} \mathrm{HNO}_{3}(2-5 \mathrm{mg}$ in $1 \mathrm{~mL})$. The samples were then diluted to $10 \mathrm{~mL}$ with deionized water and sufficient solution pipetted out that $1 \mu \mathrm{g}$ of $\mathrm{Pb}$ was available for analysis (usually about $10 \mu \mathrm{L}$ ). Mixed sulfides were digested in hot ultrapure $12 \mathrm{M} \mathrm{HCl,} \mathrm{16M}$ $\mathrm{HNO}_{3}$, or aqua regia. The solution was decanted, evaporated to dryness, and converted first to the chloride medium and then to the bromide medium. Lead was isolated from other cations using anion exchange resin in the bromide medium. Ultrapure reagents were used throughout the procedure. Analytical blanks were less than $2.5 \mathrm{ng}$ and have a negligible impact on the reported $\mathrm{Pb}$ isotope ratios. The sample was loaded on the anion exchange resin in $0.75 \mathrm{M}-1.0 \mathrm{M} \mathrm{HBr}$, washed with $1.0 \mathrm{M} \mathrm{HBr}$ and then with $1.5 \mathrm{M} \mathrm{HC1}$. The $\mathrm{Pb}$ was then eluted with $6 \mathrm{M} \mathrm{HC1}$. Molybdenite samples were prepared by digestion in hot ultrapure $6 \mathrm{M} \mathrm{HC1}$. A white precipitate, probably $\mathrm{Mo}_{3} \mathrm{Cl}_{4}(\mathrm{OH})_{2} \cdot 2 \mathrm{H}_{2} \mathrm{O}$, formed and the $\mathrm{Pb}$ remained in solution. The solution was decanted, evaporated to dryness, and converted to the $\mathrm{HBr}$ medium. Lead was purified by anion exchange in the bromide medium. Sulfide samples containing high concentrations of Sb-bearing sulfides required special preparation because $\mathrm{Sb}$ is also adsorbed on the anion exchange resin (AG-1) in the bromide medium. These samples (tetrahedrite-bearing samples) were digested in ultrapure $12 \mathrm{M} \mathrm{HC1}$ in quartz beakers. The sample was then heated to dryness driving off much of the volatile $\mathrm{SbCl}_{3}$ and leaving $\mathrm{Pb}$ in the residue. The sample residue was dissolved in $\mathrm{HBr}$ and final separation of the $\mathrm{Pb}$ was done by anion exchange in the bromide medium. Rarely was it necessary to electroplate the $\mathrm{Pb}$ samples obtained from the column-separation procedure prior to mass spectrometric analysis, however, when necessary, electro-deposition on a platinum anode was used.

\section{Mass Spectrometer}

The isotopic composition of $\mathrm{Pb}$ determined at the U.S. Geological Survey, Denver, Colo. was determined using a $30.5 \mathrm{~cm}, 68^{\circ}$ sector, solid-source mass spectrometer of NBS (National Bureau of Standards) design. This instrument was completely rebuilt by W.A. Bowman at the National Bureau of 
Standards. Samples were analyzed by Jerry Gaccetta (JG, 1987-1989) and Robert Vaughn (RV, 19911995) using the single Re-filament, silica-gel emitter technique at $1250 \pm 20^{\circ} \mathrm{C}$ (Cameron and others, 1969). A minimum of two sets of eight ratio pairs for ${ }^{206} \mathrm{~Pb} /{ }^{204} \mathrm{~Pb}$ and one set each of eight ratio pairs for ${ }^{207} \mathrm{~Pb} /{ }^{206} \mathrm{~Pb}$ and for ${ }^{208} \mathrm{~Pb} /{ }^{206} \mathrm{~Pb}$ were taken over a period of 30 to 40 minutes in a typical analysis using peak-hopping by controlling the magnetic field (Bigelow and others, 1999). A second set of $\mathrm{Pb}$ isotope data also was taken if the ion beam remained stable to improve analytical statistics.

\section{Results}

The analytical data are summarized in tables $1-4$. The data in all four tables are organized in the same manner. Data are first ordered by mineral deposit type; second, alphabetically by deposit name; and finally by sample number. A few samples were analyzed more than once to evaluate long-term reproducibility of the data. Table 1 presents the $\mathrm{Pb}$ isotope results from deposits north of the Columbia River Basalt field in Washington to the Canadian border. One sample from the Lynx pit on Vancouver Island was also analyzed and is included in this data table. Table 2 presents the $\mathrm{Pb}$ isotope results from the Blue Mountains in central and eastern Oregon and western Idaho. Table 3 presents the $\mathrm{Pb}$ isotope results from the Klamath Mountains in western Oregon and northern California. Table 4 presents the $\mathrm{Pb}$ isotope results from the Shasta mining district and from the California foothills belt in northern and central California. Table 5 summarizes the abbreviations used in the data tables to convey the maximum amount of information using the least amount of space. 
Table 1. Compilation of geologic, mineral deposit type, sample mineralogy, $\mathrm{U}, \mathrm{Th}$, and $\mathrm{Pb}$ concentrations, and $\mathrm{Pb}$ isotope data for samples from northern Washington.

\begin{tabular}{|c|c|c|c|c|c|c|c|c|c|c|c|c|c|}
\hline \multirow[b]{2}{*}{ Deposit Name } & \multirow[b]{2}{*}{ Sample No. } & \multirow[b]{2}{*}{$\begin{array}{l}\text { Host } \\
\text { Age }\end{array}$} & \multirow[b]{2}{*}{$\begin{array}{l}\text { Dep. } \\
\text { Type }\end{array}$} & \multirow[b]{2}{*}{$\begin{array}{l}\text { Latitude } \\
\text { DMS }\end{array}$} & \multirow[b]{2}{*}{$\begin{array}{l}\text { Longitude } \\
\text { DMS }\end{array}$} & \multicolumn{3}{|c|}{ Pb isotope ratios } & \multirow{2}{*}{$\begin{array}{c}\mathrm{Pb} \\
\text { Conc } \\
\text { ppm } \\
\end{array}$} & \multirow{2}{*}{$\begin{array}{c}\mathrm{U} \\
\text { Conc } \\
\text { ppm }\end{array}$} & \multirow{2}{*}{$\begin{array}{c}\text { Th } \\
\text { Conc } \\
\text { ppm }\end{array}$} & \multirow[b]{2}{*}{ Mineralogy } & \multirow{2}{*}{$\begin{array}{c}\text { Mass } \\
\text { Spectrometry } \\
\text { Analyst }\end{array}$} \\
\hline & & & & & & $\frac{{ }^{206} \mathrm{~Pb}}{{ }^{204} \mathrm{~Pb}}$ & $\frac{{ }^{207} \mathrm{~Pb}}{{ }^{204} \mathrm{~Pb}}$ & $\frac{{ }^{208} \mathrm{~Pb}}{{ }^{204} \mathrm{~Pb}}$ & & & & & \\
\hline Belcher & OD18859 & $\mathrm{K}-\mathrm{T}$ & $\mathrm{Au}-\mathrm{SKN}$ & 484326 & 1183246 & 19.478 & 15.650 & 38.474 & 12 & 3.3 & 0.007 & py & $\mathrm{JG}$ \\
\hline Cooke Mountain & OD18878 & $\mathrm{K}-\mathrm{T}$ & $\mathrm{Au}-\mathrm{SKN}$ & 484227 & 1183300 & 21.900 & 15.788 & 38.581 & 2.5 & 3.1 & 0.13 & py & JG \\
\hline Big Iron Mountain & OD18865 & $\mathrm{K}-\mathrm{T}$ & $\mathrm{Au}-\mathrm{SKN}$ & 483334 & 1183552 & 18.921 & 15.635 & 38.797 & 5.2 & 0.20 & 0.021 & py & $\mathrm{JG}$ \\
\hline Republic, Knob Hill ore & GP9-N112 & $\mathrm{K}-\mathrm{T}$ & $\mathrm{Au}-\mathrm{SKN}$ & 484005 & 1184455 & 18.526 & 15.616 & 38.784 & 3,000 & -- & -- & py & $\mathrm{JG}$ \\
\hline Republic, Knob Hill ore & EB GP-2 & $\mathrm{K}-\mathrm{T}$ & $\mathrm{Au}-\mathrm{SKN}$ & 484005 & 1184455 & 18.652 & 15.600 & 38.744 & 100 & -- & -- & py & $\mathrm{JG}$ \\
\hline Aslhend Hill & 89WASAH & -- & MVT & 484230 & 1175330 & 18.493 & 15.693 & 38.257 & na & -- & -- & $\mathrm{Gn}$ & $\mathrm{JG}$ \\
\hline Cedar Creek & BCC-1-165 & $\mathrm{D}$ & MVT & 484751 & 1172653 & 19.396 & 15.763 & 39.733 & na & -- & -- & Gn & $\mathrm{JG}$ \\
\hline Cedar Creek & BCC-1-119 & $\mathrm{D}$ & MVT & 484751 & 1172653 & 19.364 & 15.748 & 39.682 & na & -- & -- & Gn & $\mathrm{JG}$ \\
\hline Cedar Creek & BCC-5-80 & $\mathrm{D}$ & MVT & 484751 & 1172653 & 19.368 & 15.747 & 39.736 & na & -- & -- & Gn, sl & $\mathrm{JG}$ \\
\hline Hubbard (stratiform ore) & 89WAHB-1 & $\mathrm{D}$ & MVT & 485516 & 1175202 & 19.830 & 15.740 & 39.592 & na & -- & -- & Gn & $\mathrm{JG}$ \\
\hline Hubbard (vein ore) & 89WAHV-1 & $\mathrm{D}$ & MVT & 485516 & 1175202 & 19.856 & 15.749 & 39.625 & na & -- & -- & $\mathrm{Gn}$ & $\mathrm{JG}$ \\
\hline \multicolumn{14}{|l|}{ Pend Oreille Mine } \\
\hline Yellowhead ore zone & 92ABYH-1 & $1 \mathrm{C}$ & MVT & 4853 & 11722 & 19.694 & 15.814 & 40.348 & 1,000 & -- & -- & sl, gn & $\mathrm{RV}$ \\
\hline Yellowhead ore zone & 92ABYH-3 & $\mathrm{C}-\mathrm{O}$ & MVT & 4853 & 11722 & 19.762 & 15.820 & 40.424 & 1,220 & -- & -- & sl, gn & $\mathrm{RV}$ \\
\hline Josephine ore zone & 92ABJS-1 & $\mathrm{mO}$ & MVT & 4853 & 11722 & 19.520 & 15.793 & 39.987 & 2,500 & -- & -- & sl, gn & $\mathrm{RV}$ \\
\hline Josephine ore zone & 92ABJS-2 & $\mathrm{mO}$ & MVT & 4853 & 11722 & 19.552 & 15.992 & 40.064 & 2,500 & -- & -- & sl, gn & $\mathrm{RV}$ \\
\hline Josephine ore zone & $92 \mathrm{ABJS}-3$ & $\mathrm{mO}$ & MVT & 4853 & 11722 & 19.507 & 15.793 & 39.948 & 930 & -- & -- & sl, gn & RV \\
\hline Josephine ore zone & 92ABJS-4 & $\mathrm{mO}$ & MVT & 4853 & 11722 & 19.462 & 15.855 & 40.063 & 1,830 & -- & -- & sl, gn & $\mathrm{RV}$ \\
\hline Josephine ore zone & 92ABJS-5 & $\mathrm{mO}$ & MVT & 4853 & 11722 & 19.393 & 15.784 & 39.810 & 2,000 & -- & -- & sl, gn & $\mathrm{RV}$ \\
\hline Alder Mine & 86WAAL-4 & $\mathrm{J}$ & VMS & 481910 & 1200950 & 18.819 & 15.602 & 38.460 & 2,200 & -- & -- & py, cp & JG \\
\hline Blue Mountain prospect & GP3418 & $\mathrm{J}$ & VMS & 474842 & 1212812 & 18.888 & 15.605 & 38.648 & 30 & -- & -- & $\mathrm{cp}$ & MD \\
\hline Bonanza & 89WABZ-1 & $\mathrm{D} / \mathrm{C}$ & VMS & 484331 & 1174710 & 18.326 & 15.682 & 38.311 & na & -- & -- & $\mathrm{Gn}$ & $\mathrm{JG}$ \\
\hline Bonanza & 89WABZ-1 dup & $\mathrm{D} / \mathrm{C}$ & VMS & 484331 & 1174710 & 18.339 & 15.700 & 38.359 & na & -- & -- & Gn & $\mathrm{JG}$ \\
\hline California Mine & 86WACL-4 & $\mathrm{J}$ & VMS & 483610 & 1183450 & 18.974 & 15.696 & 38.646 & na & -- & -- & py, cp, Gn & JG \\
\hline Copper World extension & 86WACWE-3 & $\mathrm{P}-\mathrm{Tr}$ & VMS & 485110 & 1193530 & 18.160 & 15.540 & 37.793 & 90 & 0.065 & 0.34 & py, cp, po & JG \\
\hline Empire Creek & DDH3-293 & $\mathrm{P}-\mathrm{Tr}$ & VMS/HV? & 484915 & 1184130 & 18.855 & 15.623 & 38.409 & 1,200 & -- & -- & sl, gn, py & $\mathrm{JG}$ \\
\hline Glacier (Midas) & 1492 & $1 \mathrm{~J}-\mathrm{eK}$ & VMS & 485330 & 1215500 & 18.301 & 15.531 & 37.925 & na & -- & -- & py, cp, Gn & $\mathrm{JG}$ \\
\hline Great Excelsior & 86WAEX-3 & $\mathrm{J}$ & VMS & 485350 & 1214850 & 18.767 & 15.600 & 38.318 & 3.1 & 0.024 & 0.001 & py & JG \\
\hline Holden & GP3390 & $1 \mathrm{Tr}$ & VMS & 481145 & 1204650 & 18.366 & 15.566 & 38.056 & 300 & -- & -- & py & MD \\
\hline Holden & Holden-GN & $1 \mathrm{Tr}$ & VMS & 481145 & 1204650 & 18.312 & 15.542 & 37.974 & na & -- & -- & Gn & MD \\
\hline Holden & OD6609 & $1 \mathrm{Tr}$ & VMS & 481145 & 1204650 & 18.380 & 15.557 & 38.025 & 10 & 0.010 & 0.070 & sl & $\mathrm{JG}$ \\
\hline Lockwood pyrite & 85WALW-4 & $\mathrm{J}$ & VMS & 475745 & 1214300 & 18.315 & 15.548 & 38.044 & 35 & -- & -- & py & MD \\
\hline Lone Star (Attwood) & 86WALS-1 & $\mathrm{J}$ & VMS & 485940 & 1183615 & 19.346 & 15.660 & 38.670 & 36 & 12 & 0.92 & py, cp, sl & JG \\
\hline Lone Star (Attwood) & DDH7-298 & $\mathrm{J}$ & VMS & 485940 & 1183615 & 19.110 & 15.635 & 38.546 & 60 & 0.49 & 0.020 & $\mathrm{cp}$ & $\mathrm{JG}$ \\
\hline Longstreet Mine & 86WALST-1 & $\mathrm{O} ?$ & VMS & 481410 & 1181145 & 19.383 & 15.697 & 39.236 & 11 & 0.49 & 0.020 & $\mathrm{tt}$ & $\mathrm{JG}$ \\
\hline Mary Green & GP3388 & $\mathrm{P}$ & VMS & 481425 & 1204910 & 18.608 & 15.608 & 38.274 & 20 & -- & -- & py & MD \\
\hline Merchant Mine & GP3378RB & $\mathrm{J}$ & VMS & 474929 & 1212616 & 18.958 & 15.604 & 38.688 & na & -- & -- & asp, Gn & MD \\
\hline Western Mines (Lynx Pit) & WM-1 & $\mathrm{P}$ & VMS & 493450 & 1253635 & 18.543 & 15.581 & 38.213 & na & -- & -- & Gn, cp, py & MD \\
\hline Western Mines (Lynx Pit) & WM-1 dup & $\mathrm{P}$ & VMS & 493450 & 1253635 & 18.549 & 15.565 & 38.147 & na & -- & -- & Gn & $\mathrm{MD}$ \\
\hline
\end{tabular}




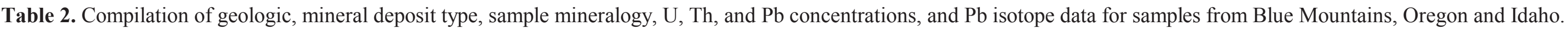

\begin{tabular}{|c|c|c|c|c|c|c|c|c|c|c|c|c|c|}
\hline \multirow[b]{2}{*}{ Deposit Name } & \multirow[b]{2}{*}{ Sample No. } & \multirow[b]{2}{*}{$\begin{array}{c}\text { Host } \\
\text { Age }\end{array}$} & \multirow[b]{2}{*}{$\begin{array}{l}\text { Dep. } \\
\text { Type }\end{array}$} & \multirow[b]{2}{*}{$\begin{array}{l}\text { Latitude } \\
\text { DMS }\end{array}$} & \multirow[b]{2}{*}{$\begin{array}{l}\text { Longitude } \\
\text { DMS }\end{array}$} & \multicolumn{3}{|c|}{$\mathrm{Pb}$ isotope ratios } & \multirow{2}{*}{$\begin{array}{c}\mathrm{Pb} \\
\text { Conc } \\
\text { ppm }\end{array}$} & \multirow{2}{*}{$\begin{array}{c}\mathrm{U} \\
\text { Conc } \\
\text { ppm }\end{array}$} & \multirow{2}{*}{$\begin{array}{c}\text { Th } \\
\text { Conc } \\
\text { ppm }\end{array}$} & \multirow[b]{2}{*}{ Mineralogy } & \multirow{2}{*}{$\begin{array}{c}\text { Mass } \\
\text { Spectrometry } \\
\text { Analyst }\end{array}$} \\
\hline & & & & & & $\frac{{ }^{206} \mathrm{~Pb}}{{ }^{204} \mathrm{~Pb}}$ & $\frac{{ }^{207} \mathrm{~Pb}}{{ }^{204} \mathrm{~Pb}}$ & $\frac{{ }^{208} \mathrm{~Pb}}{{ }^{204} \mathrm{~Pb}}$ & & & & & \\
\hline Bay Horse Mine & 89ORBH-2 & $\mathrm{mTr}$ & $\mathrm{HV}$ & 442657 & 1171311 & 18.694 & 15.588 & 38.325 & na & -- & -- & TT & $\mathrm{JG}$ \\
\hline Bay Horse Mine & 89ORBH-2 dup & $\mathrm{mTr}$ & $\mathrm{HV}$ & 442657 & 1171311 & 18.705 & 15.592 & 38.355 & na & -- & -- & $\mathrm{TT}$ & JG \\
\hline Bay Horse Mine & $\mathrm{BH}-3$ & $\mathrm{mTr}$ & $\mathrm{HV}$ & 442657 & 1171311 & 18.679 & 15.590 & 38.354 & na & -- & -- & $\mathrm{TT}$ & $\mathrm{JG}$ \\
\hline Blue Jacket & BJ-5-81 & $\mathrm{mTr}$ & VMS & 453325 & 1162610 & 18.350 & 15.528 & 37.917 & na & -- & -- & sl, cp, py, Gn & $\mathrm{JG}$ \\
\hline Blue Jacket & SR-346 & $\mathrm{mTr}$ & VMS & 453325 & 1162610 & 18.402 & 15.565 & 37.995 & 25 & -- & -- & cp, sl, gn & JG \\
\hline Buckeye Mine & 86ORBY-8 & -- & HV & 445921 & 1175145 & 18.258 & 15.518 & 37.698 & 140 & 0.77 & 0.63 & py & JG \\
\hline Clover Creek Copper & CCR-8 & $\mathrm{mTr}$ & VMS & 445435 & 1173205 & 18.310 & 15.576 & 37.942 & 210 & -- & -- & ba & $\mathrm{JG}$ \\
\hline Clover Creek Copper & OD11140 & $1 \mathrm{Tr}$ & VMS & 445435 & 1173205 & 18.776 & 15.657 & 38.546 & 25 & -- & -- & py & JG \\
\hline Copper Dyke, $100 \mathrm{ft}$ level & OD17098 & -- & VMS & 443957 & 1182923 & 18.915 & 15.694 & 34.400 & 450 & 4.6 & 0.030 & py & JG \\
\hline Dewey (Patrick Tunnel) & $3-2121 R$ & eP & VMS & 455435 & 1160145 & 18.177 & 15.538 & 37.703 & na & -- & -- & $\mathrm{Gn}, \mathrm{sl}, \mathrm{cp}$ & JG \\
\hline Dewey (Patrick Tunnel) & 89IDD-1 & $\mathrm{eP}$ & VMS & 455435 & 1160145 & 18.194 & 15.535 & 37.629 & 7.8 & 0.010 & 0.070 & py & JG \\
\hline Dolly Varden & DDH1-35 & $\mathrm{mTr}$ & VMS & 445603 & 1172214 & 18.197 & 15.501 & 37.605 & 28 & 0.15 & 0.10 & py & $\mathrm{JG}$ \\
\hline Dolly Varden & DDH1-40 & $\mathrm{mTr}$ & VMS & 445603 & 1172214 & 18.251 & 15.565 & 37.752 & 110 & 0.39 & 0.040 & py & $\mathrm{JG}$ \\
\hline Dolly Varden & DDH4-65 & $\mathrm{mTr}$ & VMS & 445603 & 1172214 & 18.206 & 15.521 & 37.663 & 51 & 0.12 & 0.030 & py & JG \\
\hline Dolly Varden & DDH4-70 & $\mathrm{mTr}$ & VMS & 445603 & 1172214 & 18.177 & 15.500 & 37.593 & 58 & 0.12 & 0.010 & py & $\mathrm{JG}$ \\
\hline Dolly Varden & DDH6-100 & $\mathrm{mTr}$ & VMS & 445603 & 1172214 & 18.428 & 15.551 & 37.878 & 7.2 & 0.030 & 0.010 & py & JG \\
\hline Dolly Varden & DDH6-190 & $\mathrm{mTr}$ & VMS & 445603 & 1172214 & 18.413 & 15.527 & 37.796 & 4.7 & 0.030 & 0.070 & py & $\mathrm{JG}$ \\
\hline Dolly Varden & DDH6-200 & $\mathrm{mTr}$ & VMS & 445603 & 1172214 & 18.306 & 15.512 & 37.694 & 7.7 & 0.030 & 0.060 & py & JG \\
\hline Dolly Varden & DDH7-100 & $\mathrm{mTr}$ & VMS & 445603 & 1172214 & 18.196 & 15.509 & 37.628 & 55 & 0.060 & 0.060 & py & JG \\
\hline Dolly Varden & DDH8-50 & $\mathrm{mTr}$ & VMS & 445603 & 1172214 & 18.180 & 15.509 & 37.625 & 170 & 0.070 & 0.040 & py & $\mathrm{JG}$ \\
\hline Dolly Varden & DDH8-55 & $\mathrm{mTr}$ & VMS & 445603 & 1172214 & 18.283 & 15.538 & 37.725 & 7.1 & 0.060 & 0.060 & py & JG \\
\hline Hercules (Cuddy Mountain) & 86IDHER-1 & $\mathrm{mTr}$ & $\mathrm{HV}$ & 444605 & 1165200 & 18.704 & 15.589 & 38.353 & 200 & -- & -- & gn & $\mathrm{JG}$ \\
\hline Hercules (Cuddy Mountain) & 86IDHER-1 dup & $\mathrm{mTr}$ & $\mathrm{HV}$ & 444605 & 1165200 & 18.705 & 15.592 & 38.355 & na & -- & -- & Gn & JG \\
\hline Idaho prospect & 89IDRQP & eP & VMS & 450045 & 1164730 & 18.292 & 15.507 & 37.710 & 5.8 & 0.13 & 0.18 & ml, py & JG \\
\hline Imperial Mine, Eagle vein & Imp-Eag & -- & $\mathrm{HV}$ & 445200 & 1181520 & 18.692 & 15.607 & 38.432 & na & -- & -- & Gn, cp, sl, py & JG \\
\hline Imperial Mine, Eagle vein & OD2023 & -- & $\mathrm{HV}$ & 445200 & 1181520 & 18.697 & 15.608 & 38.406 & na & -- & -- & Gn, cp, sl, asp, py & RV \\
\hline Imperial Mine, Eagle vein & OD2023 dup & -- & $\mathrm{HV}$ & 445200 & 1181520 & 18.687 & 15.597 & 38.383 & na & -- & -- & Gn, cp, sl, asp, py & RV \\
\hline Iron Dyke Mine & IDC-122 & eP & VMS & 450137 & 1165127 & 18.212 & 15.521 & 37.710 & na & -- & -- & Gn, sl, cp, py, tt, ba & $\mathrm{JG}$ \\
\hline Mineral & 86IDMIN-1 & $\mathrm{mTr}$ & HV & 443410 & 1170410 & 18.729 & 15.628 & 38.446 & 30 & -- & -- & $\mathrm{tt}$ & JG \\
\hline Mother Lode (Keating dist.) & BC-3 & $\mathrm{mTr}$ & VMS & 445500 & 1172838 & 18.302 & 15.528 & 37.758 & 630 & -- & -- & py, cp, sl & $\mathrm{JG}$ \\
\hline Mother Lode (Keating dist.) & BC-9 & $\mathrm{mTr}$ & VMS & 445500 & 1172838 & 18.633 & 15.674 & 38.253 & 3.6 & 0.018 & 0.018 & py & $\mathrm{JG}$ \\
\hline Oregon King Mine & OKM-1 & $\mathrm{T}$ & $\mathrm{HV}$ & 444509 & 1204419 & 19.002 & 15.608 & 38.702 & na & -- & -- & Gn, sl, cp & MD \\
\hline Overland & OD2032 & -- & $\mathrm{HV}$ & 445153 & 1181643 & 18.724 & 15.632 & 38.475 & na & -- & -- & Gn, st & $\mathrm{JG}$ \\
\hline Peck Mountain & PM-1-992 & $\mathrm{mTr}$ & VMS & 445300 & 1163600 & 18.672 & 15.603 & 38.381 & 100 & 2.1 & 0.55 & $\mathrm{cp}$ & $\mathrm{JG}$ \\
\hline Red Jacket, Union vein, level & COD6249 & -- & HV & 445930 & 1171145 & 18.628 & 15.595 & 38.221 & 560 & -- & -- & gn, cp & $\mathrm{JG}$ \\
\hline Red Ledge & TG-12-1438 & eP & VMS & 451344 & 1164007 & 18.229 & 15.518 & 37.753 & na & -- & -- & Gn, sl, py, tt, ba & $\mathrm{ALH}$ \\
\hline River Queen & OD6231 & -- & VMS & 4503 & 11647 & 18.610 & 15.588 & 38.261 & 5.0 & 0.008 & 0.007 & $\mathrm{cp}, \mathrm{tt}$ & JG \\
\hline River Queen prospect & 89IDRQP & eP & VMS & 450050 & 1164640 & 18.289 & 15.545 & 37.710 & 900 & 0.130 & 0.180 & $\mathrm{cp}$ & $\mathrm{JG}$ \\
\hline River Queen prospect & 89IDRQP dup & eP & VMS & 450050 & 1164640 & 18.298 & 15.545 & 37.791 & 900 & 0.130 & 0.180 & $\mathrm{cp}$ & JG \\
\hline Thorne Flat & TF-1 & eP & VMS & 450039 & 1165101 & 18.197 & 15.544 & 37.733 & 24 & 0.021 & 0.026 & py & $\mathrm{JG}$ \\
\hline
\end{tabular}




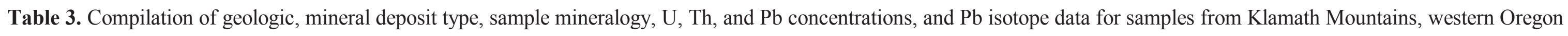
and northern California.

\begin{tabular}{|c|c|c|c|c|c|c|c|c|c|c|c|c|c|}
\hline \multirow[b]{2}{*}{ Deposit Name } & \multirow[b]{2}{*}{ Sample No. } & \multirow[b]{2}{*}{$\begin{array}{l}\text { Host } \\
\text { Age }\end{array}$} & \multirow[b]{2}{*}{$\begin{array}{l}\text { Dep. } \\
\text { Type }\end{array}$} & \multirow[b]{2}{*}{$\begin{array}{l}\text { Latitude } \\
\text { DMS }\end{array}$} & \multirow[b]{2}{*}{$\begin{array}{l}\text { Longitude } \\
\text { DMS }\end{array}$} & \multicolumn{3}{|c|}{$\mathrm{Pb}$ isotope ratios } & \multirow{2}{*}{$\begin{array}{c}\mathrm{Pb} \\
\text { Conc } \\
\text { ppm } \\
\end{array}$} & \multirow{2}{*}{$\begin{array}{c}\mathrm{U} \\
\text { Conc } \\
\text { ppm }\end{array}$} & \multirow{2}{*}{$\begin{array}{c}\text { Th } \\
\text { Conc } \\
\text { ppm } \\
\end{array}$} & \multirow[b]{2}{*}{ Mineralogy } & \multirow{2}{*}{$\begin{array}{c}\text { Mass } \\
\text { Spectrometry } \\
\text { Analyst } \\
\end{array}$} \\
\hline & & & & & & $\frac{{ }^{206} \mathrm{~Pb}}{{ }^{204} \mathrm{~Pb}}$ & $\frac{{ }^{207} \mathrm{~Pb}}{{ }^{204} \mathrm{~Pb}}$ & $\frac{{ }^{208} \mathrm{~Pb}}{{ }^{204} \mathrm{~Pb}}$ & & & & & \\
\hline Almeda & ALM-3 & $1 \mathrm{~J}$ & VMS & 423636 & 1233503 & 18.426 & 15.553 & 38.189 & na & - & -- & py, cp, Gn & MD \\
\hline Banfield & BU-1-640 & $\operatorname{Tr}$ & VMS & 425005 & 1225555 & 18.748 & 15.634 & 38.386 & 3.0 & 0.19 & 0.008 & py, cp & $\mathrm{JG}$ \\
\hline Banfield & BU-1-739 & $\operatorname{Tr}$ & VMS & 425005 & 1225555 & 19.151 & 15.615 & 38.580 & 2.0 & 0.22 & 0.20 & py & JG \\
\hline Blue Ledge & BLU-1 & $\mathrm{J}$ & VMS & 415725 & 1230630 & 18.326 & 15.545 & 37.983 & 800 & -- & -- & py, cp, sl & $\mathrm{MD}$ \\
\hline Copper Bluff & CB-1 & -- & VMS & 41635 & 1234106 & 18.200 & 15.530 & 37.855 & 1,500 & -- & -- & py, cp & $\mathrm{MD}$ \\
\hline Copper Queen & CQD-2 & $\mathrm{J}$ & VMS & 423711 & 1232332 & 18.162 & 15.536 & 37.868 & 20 & -- & -- & po, cp & $\mathrm{MD}$ \\
\hline Cowboy & CBY-1 & $\mathrm{J}$ & VMS & 420110 & 1233650 & 18.465 & 15.589 & 38.205 & 2.4 & 0.005 & 0.005 & po & $\mathrm{JG}$ \\
\hline Empire Mine/Red Ledge & C-62402 & $1 \mathrm{~J}$ & VMS & 422900 & 1233100 & 18.181 & 15.518 & 37.835 & 132 & 0.14 & 0.17 & sl & $\mathrm{JG}$ \\
\hline Goff Mine & WB-4 & $1 \mathrm{~J}$ & VMS & 424046 & 1233336 & 18.384 & 15.556 & 38.126 & 360 & -- & -- & ba, sl & $\mathrm{JG}$ \\
\hline Goff Mine & WB-4 dup & $1 \mathrm{~J}$ & VMS & 424046 & 1233336 & 18.379 & 15.551 & 38.146 & 360 & 0.17 & 0.002 & ba, sl & $\mathrm{JG}$ \\
\hline Gold Note & GN-2 & $\mathrm{J}$ & VMS & 424002 & 1231339 & 18.522 & 15.598 & 38.326 & 12 & -- & -- & po, cp & MD \\
\hline Gray Eagle & GEU-1 & $1 \mathrm{~J}$ & VMS & 415136 & 1232220 & 19.380 & 15.647 & 38.184 & 20 & 1.7 & 0.041 & po & $\mathrm{MD}$ \\
\hline H\&R & DDH5-28 & $1 \mathrm{~J}$ & VMS & 423540 & 1233615 & 18.435 & 15.546 & 38.164 & 65 & -- & -- & $\mathrm{cp}$ & $\mathrm{JG}$ \\
\hline H\&R & DDH5-55 & $1 \mathrm{~J}$ & VMS & 423540 & 1233615 & 18.449 & 15.547 & 38.180 & 30 & -- & -- & $\mathrm{cp}$ & $\mathrm{JG}$ \\
\hline H\&R & DDH6-135 & $1 \mathrm{~J}$ & VMS & 423540 & 1233615 & 18.457 & 15.561 & 38.192 & 210 & -- & -- & $\mathrm{cp}$ & $\mathrm{JG}$ \\
\hline Island Mountain & IM-2 & -- & VMS & 400154 & 1232948 & 19.084 & 15.663 & 38.897 & 350 & -- & -- & py & MD \\
\hline Island Mountain & IM-3 & -- & VMS & 400154 & 1232948 & 19.067 & 15.657 & 38.868 & 1,500 & -- & -- & py & MD \\
\hline Lyttle & LM-1 & $\mathrm{J}$ & VMS & 420200 & 1233615 & 18.383 & 15.571 & 38.122 & 5.9 & 0.003 & 0.007 & po & $\mathrm{JG}$ \\
\hline Mammoth Lode & MM-3 & $\operatorname{Tr}$ & VMS & 424540 & 1225740 & 18.729 & 15.604 & 38.423 & 2.3 & 0.007 & 0.008 & py & $\mathrm{JG}$ \\
\hline Oak Mine & OM-1 & $\operatorname{Tr}$ & VMS & 423312 & 1231759 & 18.175 & 15.502 & 37.723 & 500 & 0.017 & 0.014 & $\mathrm{sl}, \mathrm{py}, \mathrm{cp}$ & $\mathrm{JG}$ \\
\hline Queen of Bronze & QB-LCH-C & $\mathrm{J}$ & VMS & 420301 & 1233551 & 18.337 & 15.532 & 38.014 & 8.0 & 0.004 & 0.001 & py, cp & $\mathrm{JG}$ \\
\hline Rowley & RS-11-130 & $\operatorname{Tr}$ & VMS & 424858 & 1225635 & 18.232 & 15.542 & 37.998 & 260 & -- & -- & py, sl, cp & MD \\
\hline Silver Pk, lower Umpqua adit & Lu-4-MS & $1 \mathrm{~J}$ & VMS & 425120 & 1232304 & 18.378 & 15.544 & 38.109 & 80 & -- & -- & ba, py & $\mathrm{MD}$ \\
\hline Squaw Creek & SQ-4 & $\mathrm{J}$ & VMS & 420156 & 1230454 & 18.338 & 15.555 & 38.021 & 5.8 & 0.024 & 0.013 & py & $\mathrm{JG}$ \\
\hline Steamboat & C-62418 & -- & VMS & 420503 & 1231615 & 19.102 & 15.609 & 38.814 & 160 & -- & -- & py, cp & $\mathrm{JG}$ \\
\hline Turner Albright & TAB-33-151 & $1 \mathrm{~J}$ & VMS & 420000 & 1234526 & 18.294 & 15.560 & 38.058 & 120 & -- & -- & cp, py, cal & MD \\
\hline Waldo & OD6243 & $\mathrm{J}$ & VMS & 420230 & 1233500 & 18.264 & 15.550 & 38.008 & 1.4 & 0.001 & 0.002 & $\mathrm{cp}$ & $\mathrm{JG}$ \\
\hline Waldo & WD-1 & $\mathrm{J}$ & VMS & 420230 & 1233500 & 18.516 & 15.595 & 38.253 & 2.2 & 0.008 & 0.023 & po & $\mathrm{JG}$ \\
\hline
\end{tabular}




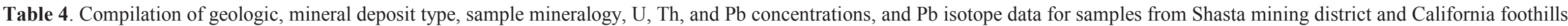
belt, California.

\begin{tabular}{|c|c|c|c|c|c|c|c|c|c|c|c|c|c|}
\hline \multirow[b]{2}{*}{ Deposit Name } & \multirow[b]{2}{*}{ Sample No. } & \multirow[b]{2}{*}{$\begin{array}{c}\text { Host } \\
\text { Age }\end{array}$} & \multirow[b]{2}{*}{$\begin{array}{l}\text { Dep. } \\
\text { Type }\end{array}$} & \multirow[b]{2}{*}{$\begin{array}{l}\text { Latitude } \\
\text { DMS }\end{array}$} & \multirow[b]{2}{*}{$\begin{array}{l}\text { Longitude } \\
\text { DMS }\end{array}$} & \multicolumn{3}{|c|}{ Pb isotope ratios } & \multirow{2}{*}{$\begin{array}{c}\text { Pb } \\
\text { Conc } \\
\text { ppm } \\
\end{array}$} & \multirow{2}{*}{$\begin{array}{c}\mathrm{U} \\
\text { Conc } \\
\text { ppm }\end{array}$} & \multirow{2}{*}{$\begin{array}{c}\text { Th } \\
\text { Conc } \\
\text { ppm } \\
\end{array}$} & \multirow[b]{2}{*}{ Mineralogy } & \multirow{2}{*}{$\begin{array}{c}\text { Mass } \\
\text { Spectrometry } \\
\text { Analyst } \\
\end{array}$} \\
\hline & & & & & & $\frac{{ }^{206} \mathrm{~Pb}}{{ }^{204} \mathrm{~Pb}}$ & $\frac{{ }^{207} \mathrm{~Pb}}{{ }^{204} \mathrm{~Pb}}$ & $\frac{{ }^{208} \mathrm{~Pb}}{{ }^{204} \mathrm{~Pb}}$ & & & & & \\
\hline Alabaster Cave & OD5415 & -- & VMS & 384843 & 1210432 & 18.356 & 15.587 & 38.135 & 6.3 & -- & -- & py, cp & RV \\
\hline Buchanan Mine & NMNH61396 & -- & VMS & 371113 & 1195927 & 18.896 & 15.616 & 38.559 & 2.5 & -- & -- & $\mathrm{cp}$ & RV \\
\hline Bully Hill & OD9054 & -- & VMS & 404745 & 1221100 & 17.980 & 15.528 & 37.642 & 380 & -- & -- & sl, cp, gn, Au, py & $\mathrm{JG}$ \\
\hline Campo Seco (Penn) & DM-5 & $1 \mathrm{~J}$ & VMS & 381318 & 1205224 & 18.284 & 15.577 & 38.079 & 290 & -- & -- & py, sl, cp, bn, tt, gn, ml & RV \\
\hline Campo Seco (Penn) & OD5566 & $1 \mathrm{~J}$ & VMS & 381318 & 1205224 & 18.302 & 15.569 & 38.075 & 6,000 & -- & -- & py, cp & RV \\
\hline Campo Seco (Penn) & OD5583 & $1 \mathrm{~J}$ & VMS & 381318 & 1205224 & 18.296 & 15.565 & 38.057 & na & -- & -- & $\mathrm{cp}, \mathrm{sl}, \mathrm{Gn}, \mathrm{cu}$ & RV \\
\hline Campo Seco (Penn) & UCB688-20 & $1 \mathrm{~J}$ & VMS & 381318 & 1205224 & 18.278 & 15.561 & 38.044 & 350 & -- & -- & $\mathrm{cp}, \mathrm{sl}, \mathrm{gn}$ & RV \\
\hline Campo Seco (Penn) & UCB688-41 & $1 \mathrm{~J}$ & VMS & 381318 & 1205224 & 18.265 & 15.538 & 37.882 & 250 & -- & -- & $\mathrm{cp}$ & RV \\
\hline Dairy Farm & OD5729 & -- & VMS & 390151 & 1211717 & 18.421 & 15.568 & 38.174 & na & -- & -- & Gn, sl, cp, py & RV \\
\hline Dairy Farm & OD5768 & -- & VMS & 390151 & 1211717 & 18.435 & 15.598 & 38.248 & 1,300 & -- & -- & py, cp & RV \\
\hline Daulton Mine & UCB160E142 & -- & VMS & 370624 & 1195801 & 18.359 & 15.562 & 38.089 & na & -- & -- & $\mathrm{Gn}, \mathrm{cp}$ & RV \\
\hline Daulton Mine & UCB160E36 & -- & VMS & 370624 & 1195801 & 18.815 & 15.660 & 38.657 & 440 & -- & -- & py, po, cp & RV \\
\hline Fresno & OD8457 & -- & VMS & 365353 & 1194005 & 18.715 & 15.548 & 37.915 & 5.4 & -- & -- & py, cp & RV \\
\hline Green Mountain Mine (Mann) & UCB160E37 & -- & VMS & 400557 & 1205635 & 18.889 & 15.690 & 38.774 & 1,400 & -- & -- & po & RV \\
\hline Ione & OD5476 & -- & VMS & 382330 & 1205703 & 18.269 & 15.568 & 38.061 & na & -- & -- & $\mathrm{cp}, \mathrm{sl}, \mathrm{Gn}$ & RV \\
\hline Jesse Bell & UCB160E212 & -- & VMS & 370826 & 1195652 & 18.869 & 15.563 & 38.314 & 1.7 & -- & -- & py, cp & RV \\
\hline Keystone-Union Mine & OD5323 & -- & VMS & 375835 & 1203834 & 18.291 & 15.541 & 37.980 & 2.4 & -- & -- & $\mathrm{cp}, \mathrm{sl}, \mathrm{gn}$ & RV \\
\hline Napoleon & OD5692 & -- & VMS & 375539 & 1204344 & 18.456 & 15.585 & 38.281 & 560 & -- & -- & $\mathrm{cp}, \mathrm{py}, \mathrm{gn}$ & RV \\
\hline Nassau & OD5626 & -- & VMS & 380240 & 1203853 & 18.180 & 15.546 & 37.909 & 490 & -- & -- & $\mathrm{cp}, \mathrm{sl}, \mathrm{gn}$ & RV \\
\hline Newton & DM-2 & $1 \mathrm{~J}$ & VMS & 382030 & 1205310 & 18.131 & 15.518 & 37.683 & 21 & -- & -- & py, cp, cc, sl & RV \\
\hline Noonday & OD5428 & -- & VMS & 383826 & 1204751 & 18.351 & 15.546 & 37.939 & 58 & -- & -- & $\mathrm{cp}, \mathrm{py}$ & RV \\
\hline Quail Hill & OD8429 & -- & VMS & 375740 & 1204457 & 18.416 & 15.561 & 38.187 & na & -- & -- & $\mathrm{cp}, \mathrm{sl}, \mathrm{Gn}$ & RV \\
\hline Valley View & DM-3 & $1 \mathrm{~J}$ & VMS & 385838 & 1211525 & 18.335 & 15.523 & 37.972 & na & -- & -- & Gn, cp, py, sl, cu & RV \\
\hline Valley View & OD5853 & $1 \mathrm{~J}$ & VMS & 385838 & 1211525 & 18.790 & 15.622 & 38.642 & na & -- & -- & sl, Gn, cp, py & RV \\
\hline
\end{tabular}


Table 5. Abbreviations used in tables $1--4$ defined.

\section{Column Heading Definition}

Deposit Name

Term used by geologist who submitted sample; value if given in parentheses is either the alternative name listed in USGS MRDS database or the mining district name if, without these data, confusion might result from the use of same deposit name in a different mining district.

Samples from museum collections are identified by suffixes on the sample numbers as follows: OD, sample from the Stanford ore deposits collection, collected with permission of M. Einaudi; UCB, University of California, Berkeley geology

Sample No. collection, collected with permission of curator; NMNH, National Museum of Natural History, collected through curator Sorena Sorensen. All other samples were collected by the author or were contributed by geologists working in mineral exploration. DDH, sample collected from diamond drill hole provided by contributor.

Host Age e, early; m, middle; or 1, late geologic time in geologic period. C, Cambrian; O, Ordovician; D, Devonian; D/C, Devonian/Carboniferous; P, Permian; Tr, Triassic; J, Jurassic; K, Cretaceous; and T, Tertiary geologic time periods.

Mineral deposit type as defined in Cox and Singer (1986); Au-SKN, gold skarn

Dep. Type deposit; MVT, Mississippi-Valley type carbonate-hosted deposit; VMS, volcanogenic-hosted massive sulfide deposit; HV, hydrothermal vein, usually polymetallic vein with or without recoverable gold.

Latitude

Longitude

DMS location expressed in degrees, minutes, seconds (NAD27). Horizontal coordinate information is referenced to North American Datum 1927.

DMS location expressed in degrees, minutes, seconds (NAD27)

Concentrations expressed in ppm, that is parts per million by weight $(\mu \mathrm{g} / \mathrm{g})$, for the $\mathrm{U}, \mathrm{Th}$, and $\mathrm{Pb}$ Conc. elements $\mathrm{U}$, uranium; Th, thorium; and $\mathrm{Pb}$, lead; na, not analyzed because $\mathrm{Pb}$ concentration was high or galena or tetrahedrite was visible in sample; --, concentration not determined. Analytical blank expressed in ng, or parts per billion by weight.

Au, gold; asp, arsenopyrite; ba, barite; bn, bornite; cal, calcite; cp, chalcopyrite; cu, cubanite?; gn (Gn), galena; ml, malachite; po, pyrohotite; py, pyrite; sl, sphalerite;

Mineralogy tt (TT) tetrahedrite. Gn or TT indicates galena or tetrahedrite existed in sample in large enough crystals that they could be readily picked and were analyzed separately using different chemistry.

From 1982 through 1986, Maryse Delaveux (MD) performed most of the analyses; Mass Spectrometry one sample was analyzed by Anne LeHuray (ALH). From 1987 through 1989, Jerry Analyst Gaccetta (JG) analyzed the samples. From 1992 through 1995 when the MRP mass spectrometry laboratory was closed, Robert Vaughn (RV) analyzed the samples. (The mass spectrometer was subsequently surplused.) 


\section{Acknowledgments}

Research in the U.S. Geological Survey is always a team effort. Certainly, I have not visited all of the deposits or occurrences from which I have reported analytical data. Many geologists have contributed samples for this compilation. I could not conduct this survey without the contributions made by many who have visited mineralized areas in the field. To them, I express my thanks for providing samples and field information for each occurrence.

\section{References Cited}

Bigelow, R.C., Vaughn, R.B., Church, S.E., 1999, MASSPEC; A PC program to control and to process data from an automated mass spectrometer: U.S. Geological Survey Open-File Report 99-161, 34 p.

Cameron, A.E., Smith, D.H., and Walker, R.L., 1969, Mass spectrometric analysis of nanogram quantities of lead: Analytical Chemistry, v. 41, p. 525-526.

Catanzaro, E.J., Murphy, T.J., Shields, W.R., and Garner, E.L., 1968, Absolute isotopic abundance ratios of common, equal-atom, and radiogenic lead isotopic standards: Journal of Research, National Bureau of Standards, v. 72A, p. 261-267.

Church, S.E., Delevaux, M.H., and Gray, J.E., 1987a, Pb-isotope data base for sulfides from Alaska, March, 1987: U.S. Geological Survey Open-File Report 87-259, 44 p.

Church, S.E., Gray, J.E., Delevaux, M.H., and LeHuray, A.P., 1987b, Lead-isotope signatures of Devonian-Mississippian massive sulfide deposits in Alaska and their significance to mineral exploration, in Elliott, I.L., and Smee, B.W., eds., GEOEXPO/86 Exploration in the North American Cordillera: Assoc. Exploration Geochemists, Ontario, Canada. p. 132-141.

Church, S.E., LeHuray, A.P., Grant, A.R., Delevaux, M.H., and Gray, J.E., 1986, Lead-isotopic data from sulfide minerals from the Cascade Range, Oregon and Washington: Geochimica Cosmochimica Acta, v, 50, p. 317-328.

Cox, D.P., and Singer, D.A., eds., 1986, Mineral deposits models: U.S. Geological Survey Bulletin $1693,379 \mathrm{p}$.

Gaccetta, J.D., and Church, S.E., 1989, Lead isotope data base for sulfides from Alaska, December, 1989: U.S. Geological Survey Open-File Report 89-688, 60 p.

Gulson, B.L., 1986, Lead isotopes in mineral exploration-Developments in economic geology: Elsevier, New York, 245 p. 